\title{
FREI, Max (2003): Begegnungen mit der Spinne.
}

Mit einem Vorwort von A. Hänggi.

127 Seiten, 38 großformatige Bildtafeln und zahlreiche schwarz-weiß Zeichnungen Format 23,5 x $32 \mathrm{~cm}$,

Ott Verlag, Thun

ISBN 3-7225-6789-0

48,- Euro

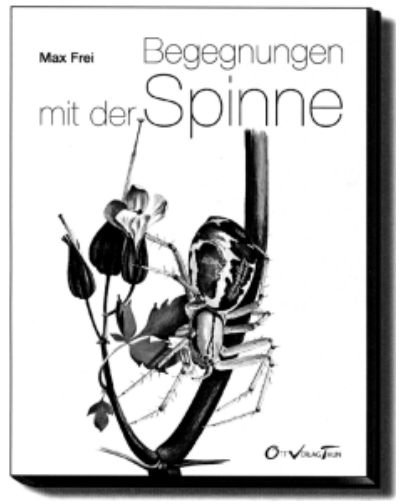

Bücher, die Begeisterung wecken, kann es für die Spinnen eigentlich nicht genug geben. Trotz der Tatsache, dass wir uns heute überall und umfassend (z.B. über das Internet) mit Informationen versorgen können, ist häufig eine allgemeine und fachlich nicht begründbare Spinnenfurcht in der Öffentlichkeit zu beobachten. Man setzt sich eben doch am liebsten mit dem auseinander, was einem liegt - oder einem nahe gebracht wird. Die Überwindung eigener Ängste kostet Energie, erst recht, wenn es sich um undifferenzierte, pauschale Ängste wie bei der Spinnenfurcht oft der Fall ist, handelt.

Da kann es sehr gut helfen, ein Buch wie das von Max Frei in den Händen zu halten: attraktive, großformatige Aufmachung, gedruckt auf hochwertigem Papier und mit einem festen Einband und farbigem Schutzumschlag versehen, dürfte das Buch vor allem (aber nicht nur!) arachnologische Laien und Einsteiger zum Lesen reizen. Auch wenn die Aquarelle nicht die Qualität eines 'Roberts' erreichen und hier und da trotz aller Liebe zum Detail wissenschaftliche Exaktheit vermissen lassen, so erfüllen sie doch mehr als ihren Zweck: Spinnen als interessante, schöne und faszinierende Lebewesen darzustellen, über die Nicht-ArachnologInnen eigentlich stets viel zu wenig wissen. 
Auch die Texte lassen aus wissenschaftlicher Sicht zu wünschen übrig aber eben weitgehend „nur“ aus wissenschaftlicher Sicht. Wir sollten lernen, ab und zu ein bisschen weniger akademisch zu sein, wenn wir unsere Tiergruppe populärer machen, ihre Akzeptanz steigern und Ängste abbauen möchten. Hochwertige wissenschaftliche Textpassagen und neue Erkenntnisse sollten versierte ArachnologInnen in anderen Büchern suchen. Das Anliegen von Max Frei ist offensichtlich: Den Mitmenschen „von nebenan" Spinnen näher zu bringen. Und dieses Ziel erreicht er. Die Texte sind anekdotenreich, z.T. regelrecht mitreißend und zeigen auch wissenschaftliche Erkenntnisse z.B. zur Evolution der Spinnen auf. Hier und da würden noch detailliertere Darstellungen $\mathrm{zu}$ einem besseren Verständnis für die nicht wissenschaftlich beschlagenen LeserInnen beitragen (z.B. zum Geschlechtsdimorphismus oder zur Giftigkeit der Spinnen).

Möge das Buch bei vielen Nicht-ArachnologInnen unter dem Weihnachtsbaum liegen - überreicht von sonst leider oft unverständlichen Fach-WissenschaftlerInnen ...

O.-D. FINCH 